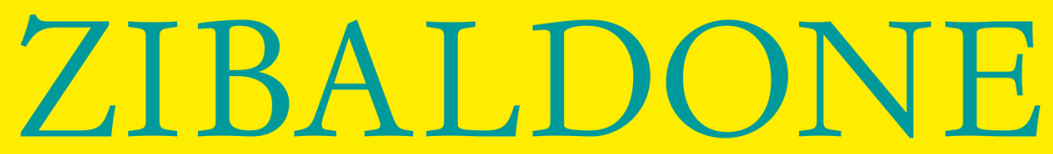

Zeitschrift für italienische Kultur der Gegenwart

No. 67

Frühjahr 2019

\title{
Sport und Gesellschaft
}

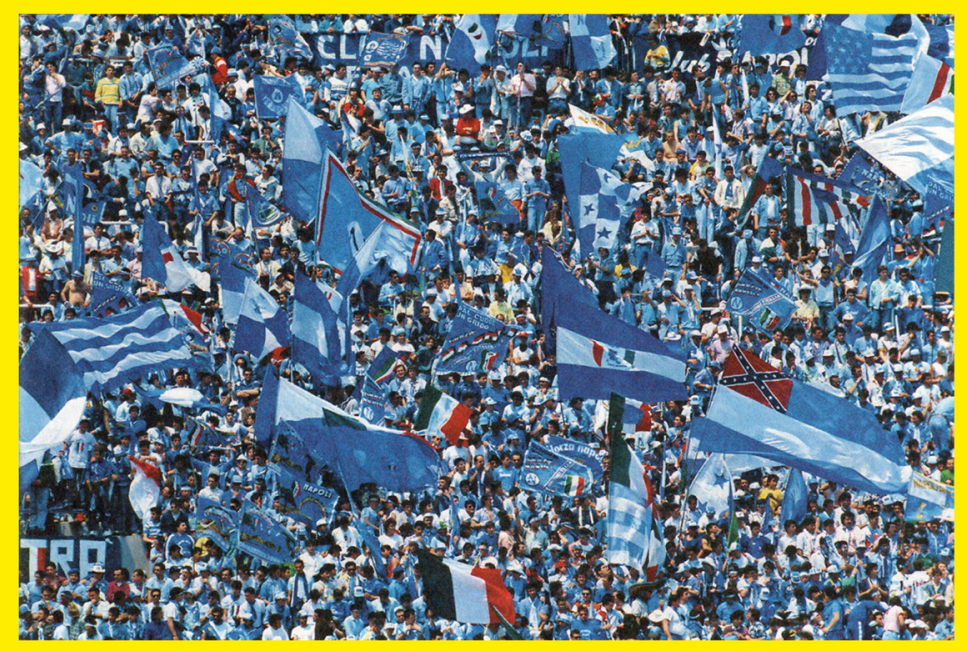

Mit Beiträgen zu
Trabrennen im Risorgimento.

sportlerinnen im
der Superga-Tragódie.
Rom 1960

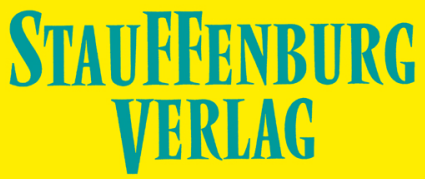




\title{
ZIBALDONE
}

Zeitschrift für italienische Kultur der Gegenwart

\author{
Begründet von
}

Titus Heydenreich und Helene Harth

\author{
Herausgegeben von \\ Thomas Bremer und Daniel Winkler
}

No. 67

Frühjahr 2019

Schwerpunkt:

Sport und Gesellschaft

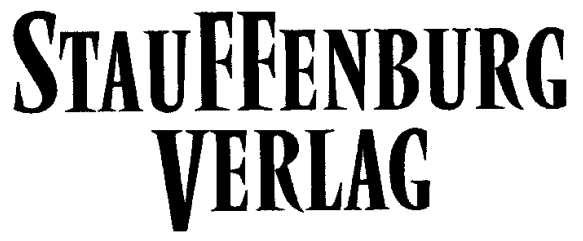


Bibliografische Information der Deutschen Bibliothek

Die Deutsche Bibliothek verzeichnet diese Publikation in der Deutschen Nationalbibliografie; detaillierte bibliografische Daten sind im Internet über <http://dnb.ddb.de> abrufbar.

Zibaldone: Zeitschrift für italienische Kultur der Gegenwart. Tübingen: Stauffenburg Verlag Bis 19 (1995) im Verl. Piper, München / Zürich

Bis 32 (2001) im Rotbuch Verl., Hamburg ISSN 0930-8997

Schwerpunkt: Sport und Gesellschaft hrsg. von Thomas Bremer und Daniel Winkler Tübingen: Stauffenburg Verlag, 2019 (Zibaldone; No. 67) ISBN 978-3-95809-710-0

Zibaldone. Zeitschrift für italienische Kultur der Gegenwart, erscheint zweimal jährlich.

Einzelhefte EUR 15,- Jahresabonnement EUR 24,- (zuzüglich Porto)

(C) 2019 Stauffenburg Verlag GmbH Postfach 2525 - D-72015 Tübingen www.stauffenburg.de

Titelbild: Napoli gegen Fiorentina im Stadio San Paolo, Mai 1987 (Foto: Roberto Koch, Quelle: wikipedia.it)

\section{Manuskripte nur an die Herausgeber:}

Prof. Dr. Thomas Bremer, Martin-Luther-Universität, Institut für Romanistik, D-06099 Halle; thomas.bremer@romanistik.uni-halle.de Ass.-Prof. Dr. Daniel Winkler, Universität Wien, Institut für Romanistik, Spitalgasse 2-Hof 8, A-1090 Wien; daniel.winkler@univie.ac.at

Sofern nicht anders angegeben, handelt es sich bei allen Beiträgen um Erstveröffentlichungen.

\section{ZIBALDONE 68}

Fiume/Rijeka. Italien und Kroatien 
Inhalt

Vorwort 7

\section{Thomas Bremer}

Ein Rennpferd als Volksheld. «Vandalo» und der Trabrenn-Sport im Risorgimento 9

Erminio Fonzo

Ondina Valla. Eine Spitzensportlerin in den Jahren des Faschismus

\section{John Foot}

Ein Erinnerungsort des italienischen Sports. Die Superga-Katastrophe von 1949 und der Mythos von Il Grande Torino 31

\section{Eva Maria Gajek}

Podest und Politik. Die Olympischen Spiele von Rom 1960

Angela Oster

Teta Veleta. Triumph und Tragödie des Fußballs bei Pier Paolo Pasolini

Sergio Giuntini

(Auto)-Biographie einer Stadt und eines Parks. Sport in Monza

Nelson Puccio

Der Radsport und seine epische Berichterstattung. Vom Entstehen und Fortbestehen eines Mythos

85

\section{Vincenzo Folino}

Sprachen und Fußball. Kommunikationsstrategien von Spielern und Trainern in mehrsprachigen Mannschaften

97

\section{Fulvia D'Aloisio}

Maradona zwischen Symbol, Mythos und globaler Dimension. Ein anthropologischer Blick auf die Fankultur des neapolitanischen Fußballs 
Notizbuch

Elisabeth Fraller über einen Reiseführer zum Jüdischen Rom - Thomas Bremer über die Escher-Ausstellung in Neapel - Interview mit Andrea D’Angelo von der Künstlerbewegung Gli indecisionisti (Janek Scholz) 121

Rezensionen 133

Zu den Autor_innen 147

\title{
Vorschau auf die Themenschwerpunkte der nächsten Hefte
}

\author{
Fiume/Rijeka. Italien und Kroatien \\ No. 68 Herbst 2019
}

Musica leggera - der Schlager in Italien

No. 69 Frühjahr 2020 


\section{ZIBALDONE 67}

\section{Sport und Gesellschaft}

Sport, sowohl aktiv als Teilnehmer_in als auch passiv als Zuschauer_in betrieben, ist eine Erscheinung, die es in den modernen Gesellschaften in größerem Umfang erst seit etwa der Wende vom 19. zum 20. Jahrhundert gibt. Ausführliche Anleitungen wie die von Giulio Franceschi erläutern beispielsweise in Italien noch 1903 die elementaren Regeln und die mittelalterliche Vorgeschichte neuerdings populär gewordener Ballspiele, vor allem des «foot ball» (das man ihm zufolge bei Bedarf gut auch mit 2x19 Teilnehmern spielen kann) und des «lawn tennis», von denen seine Leser_innen womöglich erst undeutliche Vorstellungen besitzen. Der entscheidende Durchbruch erfolgt dann in den 20er Jahren; nun gibt es auch erste Sportshelden und auch -heldinnen (unter anderem mit Ondina Valla) in einer großen Vielzahl von Sportarten, in der Leichtathletik ebenso wie im Motorsport, aber natürlich auch im in der Zwischenzeit sehr schnell weit verbreiteten Fußball und Tennis.

Mit diesem Heft möchte Zibaldone der Tradition des Sports und seiner Verbindung mit der italienischen Gesellschaftsentwicklung nachgehen und damit eine Brücke zu unmittelbar aktuellen Ereignissen schlagen, wie sie in der Tagespresse verfolgt werden können.

Wie immer, danken wir alle denjenigen, die mit Texten und ungewöhnlichen Bildern zu diesem Heft beigetragen haben, sowie denjenigen, die Kontakte her- und Bildrechte zur Verfügung gestellt haben. Allen Leserinnen und Lesern wünschen wir eine anregende Lektüre.

Die Herausgeber 